\title{
Minimal sets in singularly perturbed systems with three time-scales
}

\author{
Pedro Toniol Cardin, \\ Departamento de Matemática, FEIS, UNESP, \\ 15385-000, Ilha Solteira, SP \\ E-mail: pedrocardin@mat.feis.unesp.br, \\ Paulo Ricardo da Silva \\ Departamento de Matemática, IBILCE, UNESP, \\ 15054-000, São José do Rio Preto, SP \\ E-mail: prs@ibilce.unesp.br. \\ Marco Antonio Teixeira \\ Departamento de Matemática, IMECC, UNICAMP \\ 13081-970, Campinas, SP \\ E-mail: teixeira@ime.unicamp.br.
}

Abstract: In this work we study three time scale singular perturbation problems

$$
\varepsilon x^{\prime}=f(\mathbf{x}, \varepsilon, \delta), \quad y^{\prime}=g(\mathbf{x}, \varepsilon, \delta), \quad z^{\prime}=\delta h(\mathbf{x}, \varepsilon, \delta),
$$

where $\mathbf{x}=(x, y, z) \in \mathbb{R}^{n} \times \mathbb{R}^{m} \times \mathbb{R}^{p}, \varepsilon$ and $\delta$ are two independent small parameter $(0<\varepsilon$, $\delta \ll 1$ ), and $f, g, h$ are $C^{r}$ functions, with $r \geq 1$. We establish conditions for the existence of compact invariant sets (singular points, periodic and homoclinic orbits) when $\varepsilon, \delta>0$. Our main strategy is to consider three time scales which generate three different limit problems.

Keywords: Singular perturbations problems, three time scales

In this work we study systems with three distinct time-scales. These systems are in general written in the form

$$
\varepsilon x^{\prime}=f(\mathbf{x}, \varepsilon, \delta), \quad y^{\prime}=g(\mathbf{x}, \varepsilon, \delta), \quad z^{\prime}=\delta h(\mathbf{x}, \varepsilon, \delta),
$$

where $\mathbf{x}=(x, y, z) \in \mathbb{R}^{n} \times \mathbb{R}^{m} \times \mathbb{R}^{p}, \varepsilon$ and $\delta$ are two independent small parameter $(0<\varepsilon$, $\delta \ll 1$ ), and $f, g, h$ are $C^{r}$ functions, where $r$ is big enough for our purposes. In system (1) three different time-scales can be derived: a slow time-scale $t$, an intermediate time-scale $\tau_{1}:=\frac{t}{\delta}$ and a fast time-scale $\tau_{2}:=\frac{\tau_{1}}{\varepsilon}$.

Example. Examples of models involving three time-scales are for instance found in food chain models with a third class of so-called super or top-predators ([7], [1] and [2]) or in hormone secretion models ([5]). For instance, the Rosenzweig-MacArthur model ([8]) for tritrophic food chains (as proposed by [1])

$$
\varepsilon x^{\prime}=x\left(1-x-\frac{y}{x+b_{1}}\right), \quad y^{\prime}=y\left(\frac{x}{x+b_{1}}-d_{1}-\frac{z}{y+b_{2}}\right), \quad z^{\prime}=\delta z\left(\frac{y}{y+b_{2}}-d_{2}\right),
$$

is an example of a problem involving three different time-scales. It is composed of a logistic prey $x$, a Holling type II predator $y$ and a Holling type II top-predator $z$. Models with three or more time-scales are also used to study neuronal behavior, in particular to explain firing of 
neurons or so-called mixed mode oscillations (see [4], [6]).

In this work we develop a mathematical theory in order to study systems (1). Our main goal is to build a theory, inspired by the one given by Fenichel in [3], for systems involving three different time-scales.

\section{Statement of the main results}

The system (1) is written with respect to the time-scale $\tau_{1}$ so it is called intermediate system. By transforming (1) to the slow and fast variables $t$ and $\tau_{2}$ we obtain, respectively, the slow system

$$
\varepsilon \delta x^{\prime}=f(\mathbf{x}, \varepsilon, \delta), \quad \delta y^{\prime}=g(\mathbf{x}, \varepsilon, \delta), \quad z^{\prime}=h(\mathbf{x}, \varepsilon, \delta),
$$

and the fast system

$$
x^{\prime}=f(\mathbf{x}, \varepsilon, \delta), \quad y^{\prime}=\varepsilon g(\mathbf{x}, \varepsilon, \delta), \quad z^{\prime}=\varepsilon \delta h(\mathbf{x}, \varepsilon, \delta) .
$$

Remark. To simplify our notation, we will use the notation $x^{\prime}$ to indicate the derivative with respect to the three time scales. More specifically, for the systems (1), (3) and (4), $x^{\prime}$ indicates the derivatives $\frac{d x}{d \tau_{1}}, \frac{d x}{d t}$ and $\frac{d x}{d \tau_{2}}$, respectively.

Note that, for $\varepsilon, \delta \neq 0$, systems (1), (3) and (4) are equivalent. By setting $\varepsilon=\delta=0$ in (1), (3) and in (4) we obtain three systems with dynamics essentially different: the intermediate problem

$$
0=f(\mathbf{x}, 0,0), \quad y^{\prime}=g(\mathbf{x}, 0,0), \quad z^{\prime}=0,
$$

the reduced problem

$$
0=f(\mathbf{x}, 0,0), \quad 0=g(\mathbf{x}, 0,0), \quad z^{\prime}=h(\mathbf{x}, 0,0),
$$

and the layer problem

$$
x^{\prime}=f(\mathbf{x}, 0,0), \quad y^{\prime}=0, \quad z^{\prime}=0 .
$$

For each $\varepsilon$ and $\delta$, consider the following sets

$$
\mathcal{S}_{1}^{\delta}=\left\{\mathbf{x} \in \mathbb{R}^{n+m+p}: f(\mathbf{x}, 0, \delta)=0\right\}
$$

and

$$
\mathcal{S}_{2}^{\varepsilon}=\left\{\mathbf{x} \in \mathbb{R}^{n+m+p}: f(\mathbf{x}, \varepsilon, 0)=g(\mathbf{x}, \varepsilon, 0)=0\right\} .
$$

Note that the intermediate and reduced problems (5) and (6) are dynamical systems defined on $\mathcal{S}_{1}^{0}$ and $\mathcal{S}_{2}^{0}$, respectively. On the other hand $\mathcal{S}_{1}^{0}$ is a manifold of singular points for $(7)$. In what follows we refer to $\mathcal{S}_{1}^{0}$ and $\mathcal{S}_{2}^{0}$ as the intermediate and slow manifolds, respectively. The reason for these names is that on $\mathcal{S}_{1}^{0}$ the intermediate time-scale is dominating and on $\mathcal{S}_{2}^{0}$ the slow time-scale predominates.

Following the ideas of the geometric singular perturbation theory [3], our goal will be to prove that one can obtain information on the dynamics of the system (1), for small values of $\varepsilon$ and $\delta$, by suitably combining the dynamics of the three limit problems (5), (6) and (7).

Four other systems will also play an important role in our analysis of system (1). By setting $\varepsilon=0$ in (1) (or in (3)) and in (4) while keeping $\delta$ fixed but nonzero, we obtain the $\delta$-intermediate problem

$$
0=f(\mathbf{x}, 0, \delta), \quad y^{\prime}=g(\mathbf{x}, 0, \delta), \quad z^{\prime}=\delta h(\mathbf{x}, 0, \delta),
$$

and the $\delta$-layer problem

$$
x^{\prime}=f(\mathbf{x}, 0, \delta), \quad y^{\prime}=0, \quad z^{\prime}=0 .
$$


By setting $\delta=0$ in (1) (or in (4)) and in (3) while keeping $\varepsilon$ fixed but nonzero, we obtain the $\varepsilon$-intermediate problem

$$
\varepsilon x^{\prime}=f(\mathbf{x}, \varepsilon, 0), \quad y^{\prime}=g(\mathbf{x}, \varepsilon, 0), \quad z^{\prime}=0,
$$

and the $\varepsilon-$ reduced problem

$$
0=f(\mathbf{x}, \varepsilon, 0), \quad 0=g(\mathbf{x}, \varepsilon, 0), \quad z^{\prime}=h(\mathbf{x}, \varepsilon, 0) .
$$

Note that when both $\varepsilon, \delta \rightarrow 0$, the two $\delta, \varepsilon$-intermediate problems (8) and (10) become the same limit problem (5). The problems (8) and (11) are dynamical systems defined on the manifolds $\mathcal{S}_{1}^{\delta}$ and $\mathcal{S}_{2}^{\varepsilon}$, respectively. On the other hand, $\mathcal{S}_{1}^{\delta}$ and $\mathcal{S}_{2}^{\varepsilon}$ are sets of singular points for the problems (9) and (10), respectively.

Definition 1.1. We say that system (1) is normally hyperbolic at $\mathbf{x}_{0} \in \mathcal{S}_{2}^{0}$ if the real parts of the eigenvalues of the Jacobian matrix

$$
\left(\begin{array}{c}
D_{1,2} f\left(\mathbf{x}_{0}, 0,0\right) \\
D_{1,2} g\left(\mathbf{x}_{0}, 0,0\right)
\end{array}\right)
$$

are nonzero. We say that system (1) is $\delta$-normally hyperbolic at $\mathbf{x}_{0} \in \mathcal{S}_{1}^{\delta}$ if the real parts of the eigenvalues of the Jacobian $D_{1} f\left(\mathbf{x}_{0}, 0, \delta\right)$ are nonzero.

Now we are in position to state our main results.

Theorem A. Consider the $C^{r}$ family (1). Let $\mathcal{N} \subseteq \mathcal{S}_{2}^{0}$ be a j-dimensional compact normally hyperbolic invariant manifold of the reduced problem (6) Then there are $\varepsilon_{1}>0$ and $\delta_{1}>0$ and a $C^{r-1}$ family of manifolds $\left\{\mathcal{N}_{\delta}^{\varepsilon}: \delta \in\left(0, \delta_{1}\right), \varepsilon \in\left(0, \varepsilon_{1}\right)\right\}$ such that $\mathcal{N}_{0}^{0}=\mathcal{N}$ and $\mathcal{N}_{\delta}^{\varepsilon}$ is a hyperbolic invariant manifold of (1).

Proof. Firstly we use Fenichel's first theorem to study the persistence of $\mathcal{N}$ under $\delta$-perturbations of the system (8). Fenichel's first theorem states that the compact normally hyperbolic invariant manifold $\mathcal{N}$ of the reduced problem (6) persists, for $\delta \neq 0$ small, as an invariant manifold $\mathcal{N}_{\delta}$ for the system (8). More precisely, there exists $\delta_{1}>0$ and a $C^{r-1}$ family of manifolds $\left\{\mathcal{N}_{\delta}: \delta \in\left(-\delta_{1}, \delta_{1}\right)\right\}$ such that $\mathcal{N}_{0}=\mathcal{N}$ and $\mathcal{N}_{\delta}$ is a hyperbolic invariant manifold of (8). Now, for each $\delta$ fixed, we use again the Fenichel's Theory to study the persistence of $\mathcal{N}_{\delta}$ under $\varepsilon^{-}$ perturbations of the system (1). Note that the system (8) corresponds to the reduced problem associated to the system (1). Fenichel's first theorem says that the compact $\delta$-normally hyperbolic invariant manifold $\mathcal{N}_{\delta}$ of (8) persists, for $\varepsilon \neq 0$ sufficiently small, for the system (1), that is, there exists $\varepsilon_{1}>0$ and a $C^{r-1}$ family of manifolds $\left\{\mathcal{N}_{\delta}^{\varepsilon}: \varepsilon \in\left(-\varepsilon_{1}, \varepsilon_{1}\right)\right\}$ such that $\mathcal{N}_{\delta}^{0}=\mathcal{N}_{\delta}$ and $\mathcal{N}_{\delta}^{\varepsilon}$ is a hyperbolic invariant manifold of (1). This complete the proof of Theorem A.

Consider system (8) supplemented by the trivial equation $\delta^{\prime}=0$

$$
0=f(\mathbf{x}, 0, \delta), \quad y^{\prime}=g(\mathbf{x}, 0, \delta), \quad z^{\prime}=\delta h(\mathbf{x}, 0, \delta), \quad \delta^{\prime}=0 .
$$

Let $G(\mathbf{x}, \delta):=(g(\mathbf{x}, 0, \delta), \delta h(\mathbf{x}, 0, \delta), 0)$ be the vector field defined by (12). Assume that the linearization of $G$ at points $(\mathbf{x}, 0)$, such that $\mathbf{x} \in \mathcal{S}_{2}^{0}$, has $k^{s}$ eigenvalues with negative real part and $k^{u}$ eigenvalues with positive real part. The corresponding stable and unstable eigenspaces have dimensions $k^{s}$ and $k^{u}$, respectively.

Similarly, consider system (4) supplemented by the trivial equation $\varepsilon^{\prime}=0$

$$
x^{\prime}=f(\mathbf{x}, \varepsilon, \delta), \quad y^{\prime}=\varepsilon g(\mathbf{x}, \varepsilon, \delta), \quad z^{\prime}=\varepsilon \delta h(\mathbf{x}, \varepsilon, \delta), \quad \varepsilon^{\prime}=0 .
$$

Let $H(\mathbf{x}, \varepsilon, \delta):=(f(\mathbf{x}, \varepsilon, \delta), \varepsilon g(\mathbf{x}, \varepsilon, \delta), \varepsilon \delta h(\mathbf{x}, \varepsilon, \delta), 0)$ be the vector field defined by (13). Assume that the linearization of $H$ at points $(\mathbf{x}, 0, \delta)$, such that $\mathbf{x} \in \mathcal{S}_{1}^{\delta}$, has $l^{s}$ and $l^{u}$ eigenvalues with 
negative and positive real parts, so that the corresponding stable and unstable eigenspaces have dimensions $l^{s}$ and $l^{u}$, respectively.

Theorem B. Under the hypotheses of Theorem A, suppose that $\mathcal{N}$ has a $\left(j+j^{s}\right)$-dimensional local stable manifold $W^{s}$ and $a\left(j+j^{u}\right)$-dimensional local unstable manifold $W^{u}$. Then there are $\varepsilon_{1}>0$ and $\delta_{1}>0$ and $C^{r-1}$ families of $\left(j+j^{s}+k^{s}+l^{s}\right)$-dimensional and $\left(j+j^{u}+k^{u}+l^{u}\right)$ dimensional manifolds $\left\{\mathcal{W}_{\delta, \varepsilon}^{s}: \delta \in\left(0, \delta_{1}\right), \varepsilon \in\left(0, \varepsilon_{1}\right)\right\}$ and $\left\{\mathcal{W}_{\delta, \varepsilon}^{u}: \delta \in\left(0, \delta_{1}\right), \varepsilon \in\left(0, \varepsilon_{1}\right)\right\}$ such that for $\delta, \varepsilon>0$ the manifolds $\left\{\mathcal{W}_{\delta, \varepsilon}^{s}\right\}$ and $\left\{\mathcal{W}_{\delta, \varepsilon}^{u}\right\}$ are local stable and unstable manifolds of $\mathcal{N}_{\delta}^{\varepsilon}$, respectively.

Proof. Fenichel's second theorem says that, for small nonzero $\delta$, the invariant manifold $\mathcal{N}_{\delta}$ of (8) has a $\left(j+j^{s}+k^{s}\right)$-dimensional local stable manifold $\mathcal{W}_{\delta}^{s}$ and a $\left(j+j^{u}+k^{u}\right)$-dimensional local unstable manifold $\mathcal{W}_{\delta}^{u}$. Now, for each $\delta$ fixed, Fenichel's second theorem also states that, for $\varepsilon \neq 0$ sufficiently small, the invariant manifold $\mathcal{N}_{\delta}^{\varepsilon}$ of $(1)$ has a $\left(j+j^{s}+k^{s}+l^{s}\right)$-dimensional local stable manifold $\mathcal{W}_{\delta, \varepsilon}^{s}$ and a $\left(j+j^{u}+k^{u}+l^{u}\right)$-dimensional local unstable manifold $\mathcal{W}_{\delta}^{u}$. This complete the proof of Theorem B.

\section{Examples}

In this section we give some examples where Theorems A and B are applied.

Example 1. Consider the following 3-dimensional system

$$
\varepsilon x^{\prime}=x-\varepsilon+\delta, \quad y^{\prime}=-y+\varepsilon+\delta, \quad z^{\prime}=\delta z .
$$

The intermediate and slow manifolds $\mathcal{S}_{1}^{0}$ and $\mathcal{S}_{2}^{0}$ are given, respectively, by $\mathcal{S}_{1}^{0}=\left\{(x, y, z) \in \mathbb{R}^{3}\right.$ : $x=0\}$ and $\mathcal{S}_{2}^{0}=\left\{(x, y, z) \in \mathbb{R}^{3}: x=y=0\right\}$. On $\mathcal{S}_{1}^{0}$ we have defined the intermediate problem

$$
0=x, \quad y^{\prime}=-y, \quad z^{\prime}=0,
$$

and on $\mathcal{S}_{2}^{0}$ we have defined the reduced problem

$$
0=x, \quad 0=y, \quad z^{\prime}=z .
$$

Moreover, the layer problem is given by

$$
x^{\prime}=x, \quad y^{\prime}=0, \quad z^{\prime}=0 .
$$

Figure 1 illustrates the phase portraits of the problems (15), (16) and (17), respectively.

By using the notation given in Theorem $\mathrm{B}$, note that we have $j=0, j^{s}=0, j^{u}=1, k^{s}=1$, $k^{u}=0, l^{s}=0$ e $l^{u}=1$. We can then apply Theorems $\mathrm{A}$ and $\mathrm{B}$ at the normally hyperbolic singular point $\mathcal{N}=(0,0,0)$ of (16). Applying Theorem $\mathrm{A}$, we obtain for small nonzero $\delta, \varepsilon$, a family $\mathcal{N}_{\delta}^{\varepsilon}$ of hyperbolic singular points of (14). In fact, the family $\mathcal{N}_{\delta}^{\varepsilon}$ of singular points is given by $(\varepsilon-\delta, \varepsilon+\delta, 0)$. Applying Theorem $\mathrm{B}$, we can conclude that each singular point $\mathcal{N}_{\delta}^{\varepsilon}$ has a 1-dimensional local stable manifold $\mathcal{W}_{\delta, \varepsilon}^{s}$ and a 2 -dimensional local unstable manifold $\mathcal{W}_{\delta, \varepsilon}^{u}$.

In the next example we study the dynamics of a biological model.

Example 2. Consider the Rosenzweig-MacArthur model ([8]) for tritrophic food chains (as 

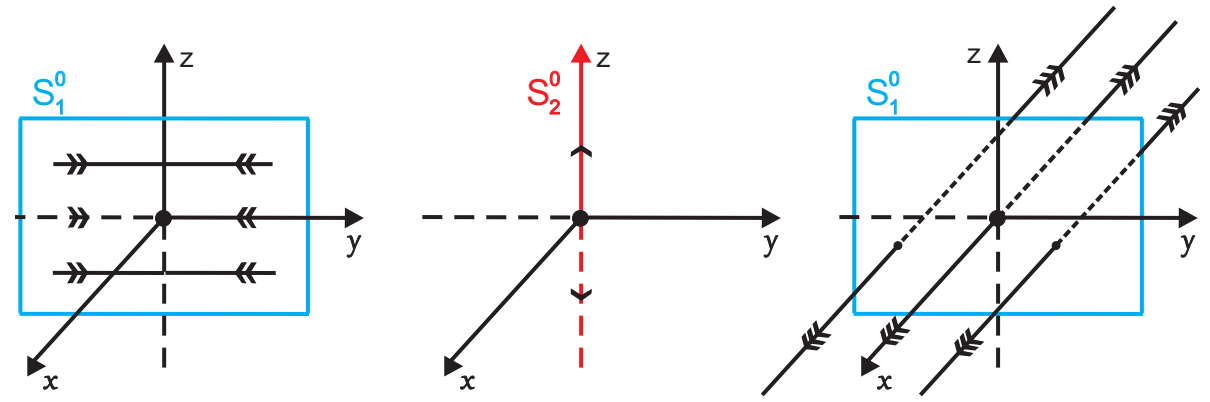

Figura 1: Phase portraits of the systems (15), (16) and (17), respectively.

proposed by [1])

$$
\begin{aligned}
\varepsilon x^{\prime} & =x\left(1-x-\frac{y}{x+b_{1}}\right)=x f(x, y), \\
y^{\prime} & =y\left(\frac{x}{x+b_{1}}-d_{1}-\frac{z}{y+b_{2}}\right)=y g(x, y, z), \\
z^{\prime} & =\delta z\left(\frac{y}{y+b_{2}}-d_{2}\right)=\delta z h(y),
\end{aligned}
$$

where $x, y$ and $z$ are 1-dimensional variables which represent a logistic prey, a Holling type II predator and a Holling type II top-predator, respectively. All parameters $b_{1}, b_{2}, d_{1}$ and $d_{2}$ are assumed to be positive and less than 1, i.e., $0<b_{1}, b_{2}, d_{1}, d_{2}<1$. We note that all discussions below are restricted to the first octant, i.e., $x \geq 0, y \geq 0$ e $z \geq 0$.

The intermediate and slow manifolds $\mathcal{S}_{1}^{0}$ and $\mathcal{S}_{2}^{0}$ are given, respectively, by $\mathcal{S}_{1}^{0}=\{x f(x, y)=$ $0\}=\{(x, y, z): x=0\} \cup\left\{(x, y, z): y=(1-x)\left(b_{1}+x\right)\right\}=M_{1} \cup M_{2}$ and $\mathcal{S}_{2}^{0}=\{x f(x, y)=$ $y g(x, y, z)=0\}=\{(x, y, z): x=y=0\} \cup\{(x, y, z): x=1, y=0\}=M_{3} \cup M_{4}$.

The intermediate problem is a dynamical system defined on $\mathcal{S}_{1}^{0}=M_{1} \cup M_{2}$. On $M_{1}$ it is given by

$$
y^{\prime}=y\left(-d_{1}-\frac{z}{y+b_{2}}\right), \quad z^{\prime}=0,
$$

and on $M_{2}$ it becomes

$$
y^{\prime}=y\left(\frac{x}{x+b_{1}}-d_{1}-\frac{z}{y+b_{2}}\right), \quad z^{\prime}=0 .
$$

The reduced problem is a dynamical system defined on $\mathcal{S}_{2}^{0}=M_{3} \cup M_{4}$. On both $M_{3}$ and $M_{4}$ it is given by

$$
z^{\prime}=-d_{2} z
$$

The layer problem is given by

$$
x^{\prime}=x\left(1-x-\frac{y}{x+b_{1}}\right), \quad y^{\prime}=0, \quad z^{\prime}=0 .
$$

Figure 2 illustrates the phase portraits of the reduced and layer problems, respectively. Figure 3 illustrates the phase portraits of the systems (19) and (20), respectively. For the phase portrait of $(20)$ we are assuming that $1 /\left(1+b_{1}\right)>d_{1}$.

Note that $\mathcal{N}=(0,0,0)$ and $\mathcal{M}=(1,0,0)$ are singular points of (21). Moreover, according with item (i) of the Definition 1.1, system (18) is normally hyperbolic at $\mathcal{N}$ and $\mathcal{M}$ (for the point $\mathcal{M}$ we are supposing that $\left.d_{1} \neq 1 /\left(1+b_{1}\right)\right)$. Applying Theorem A, we obtain for small 

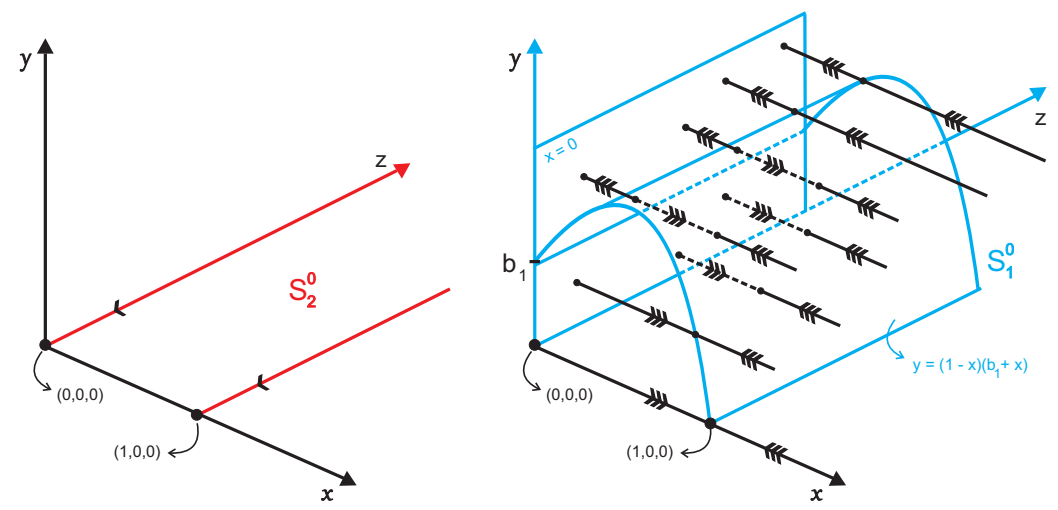

Figura 2: Phase portraits of the systems (21) and (22), respectively.
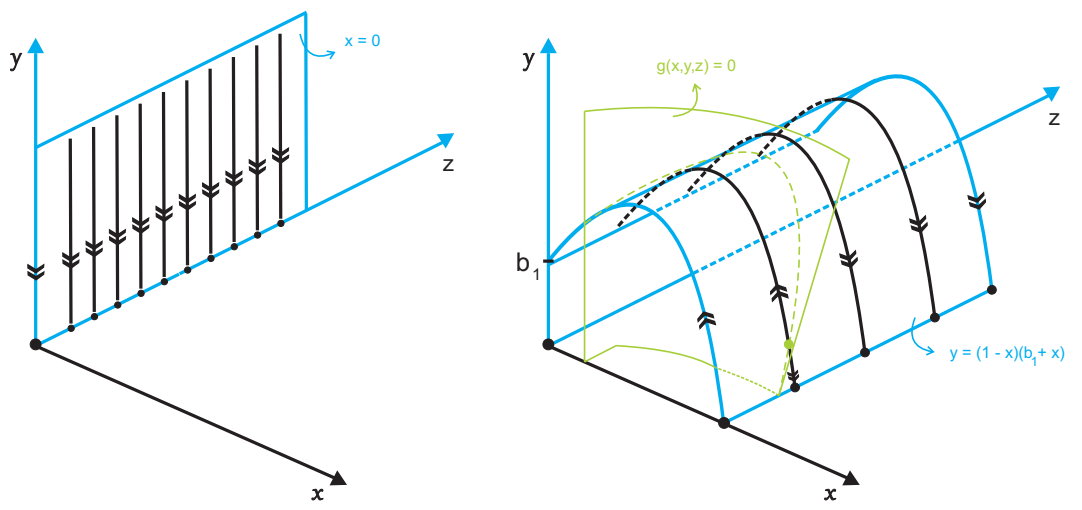

Figura 3: Phase portraits of the systems (19) and (20), respectively.

nonzero $\delta$, $\varepsilon$, families $\mathcal{N}_{\delta}^{\varepsilon}$ and $\mathcal{M}_{\delta}^{\varepsilon}$ of hyperbolic singular points of (18). In fact, the persistent singular points $\mathcal{N}_{\delta}^{\varepsilon}$ and $\mathcal{M}_{\delta}^{\varepsilon}$ are given by $(0,0,0)$ and $(1,0,0)$, respectively.

By using the notation given in Theorem B, we have that: for the point $\mathcal{N}, j=0, j^{s}=1$, $j^{u}=0, k^{s}=1, k^{u}=0, l^{s}=0$ e $l^{u}=1$, and for the point $\mathcal{M}, j=0, j^{s}=1, j^{u}=0, k^{s}=0$, $k^{u}=1, l^{s}=1$ e $l^{u}=0$. Applying Theorem B, we can conclude that each singular point $\mathcal{N}_{\delta}^{\varepsilon}$ has a 2-dimensional local stable manifold $\mathcal{W}_{\delta, \varepsilon}^{s}$ and a 1-dimensional local unstable manifold $\mathcal{W}_{\delta, \varepsilon}^{u}$. Moreover, each singular point $\mathcal{M}_{\delta}^{\varepsilon}$ has a 2 -dimensional local stable manifold $\overline{\mathcal{W}}_{\delta, \varepsilon}^{s}$ and a 1-dimensional local unstable manifold $\overline{\mathcal{W}}_{\delta, \varepsilon}^{u}$.

Example 3. Consider the following 4-dimensional system

$$
\begin{aligned}
\varepsilon x^{\prime} & =x-z_{1}+\delta+\varepsilon=f\left(x, z_{1}, \delta, \varepsilon\right), \\
y^{\prime} & =-y-z_{2}+\delta-\varepsilon=g\left(y, z_{2}, \delta, \varepsilon\right), \\
z_{1}^{\prime} & =\delta h_{1}\left(x, z_{1}, z_{2}\right), \\
z_{2}^{\prime} & =\delta h_{2}\left(y, z_{1}, z_{2}\right),
\end{aligned}
$$

where $h_{1}\left(x, z_{1}, z_{2}\right)=-z_{2}-z_{1}\left(-1+z_{1}^{2}+z_{2}^{2}\right)+\left(x-z_{1}\right)^{2}$ and $h_{2}\left(y, z_{1}, z_{2}\right)=z_{1}-z_{2}\left(-1+z_{1}^{2}+\right.$ $\left.z_{2}^{2}\right)-\left(y+z_{2}\right)^{2}$. The intermediate and slow manifolds $\mathcal{S}_{1}^{0}$ and $\mathcal{S}_{2}^{0}$ are given, respectively, by $\mathcal{S}_{1}^{0}=\left\{\left(z_{1}, y, z_{1}, z_{2}\right) \in \mathbb{R}^{4}: y, z_{1}, z_{2} \in \mathbb{R}\right\}$ and $\mathcal{S}_{2}^{0}=\left\{\left(z_{1},-z_{2}, z_{1}, z_{2}\right) \in \mathbb{R}^{4}: z_{1}, z_{2} \in \mathbb{R}\right\}$. Note that $\mathcal{S}_{1}^{0}$ and $\mathcal{S}_{2}^{0}$ are manifolds of dimension 3 and 2 , respectively.

On $\mathcal{S}_{1}^{0}$ we have defined the intermediate problem

$$
x=z_{1}, \quad y^{\prime}=-y-z_{2}, \quad z_{1}^{\prime}=0, \quad z_{2}^{\prime}=0,
$$

and on $\mathcal{S}_{2}^{0}$ we have defined the reduced problem

$$
x=z_{1}, \quad y=-z_{2}, \quad z_{1}^{\prime}=-z_{2}-z_{1}\left(-1+z_{1}^{2}+z_{2}^{2}\right), \quad z_{2}^{\prime}=z_{1}-z_{2}\left(-1+z_{1}^{2}+z_{2}^{2}\right) .
$$




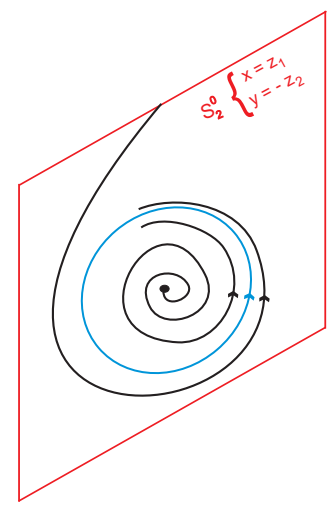

Figura 4: Phase portrait of the system (25).

Moreover, the layer problem is given by

$$
x^{\prime}=x-z_{1}, \quad y^{\prime}=0, \quad z_{1}^{\prime}=0, \quad z_{2}^{\prime}=0 .
$$

For the phase portrait of the reduced problem we can use polar coordinates $z_{1}=r \cos \theta$ and $z_{2}=r \sin \theta$. Using these coordinates it is easy to see that the system (25) presents a singular point $\mathcal{P}$ at the origin and a stable limit cycle $\Gamma$, as shown Figure 4.

According with item (i) of the Definition 1.1, all points of the slow manifold are normally hyperbolic. Applying Theorem A, we obtain for small nonzero $\delta, \varepsilon$, families $\mathcal{P}_{\delta}^{\varepsilon}$ and $\Gamma_{\delta}^{\varepsilon}$ of hyperbolic singular points and limit cycles of (23), respectively, such that $\mathcal{P}_{0}^{0}=\mathcal{P}$ and $\Gamma_{0}^{0}=\Gamma$. By using the notation given in Theorem $\mathrm{B}$, we have that: for the point $\mathcal{P}, j=0, j^{s}=0, j^{u}=2$, $k^{s}=1, k^{u}=0, l^{s}=0$ e $l^{u}=1$, and for the limit cycle $\Gamma, j=1, j^{s}=1, j^{u}=-1, k^{s}=1, k^{u}=0$, $l^{s}=0$ e $l^{u}=1$. In agreement with Theorem B, each singular point $\mathcal{P}_{\delta}^{\varepsilon}$ has an 1 -dimensional local stable manifold $\mathcal{P}_{\delta, \varepsilon}^{s}$ and a 3 -dimensional local unstable manifold $\mathcal{P}_{\delta, \varepsilon}^{u}$. Each limit cycle $\Gamma_{\delta}^{\varepsilon}$ has a 3 -dimensional local stable manifold $\Gamma_{\delta, \varepsilon}^{s}$ and an 1-dimensional local unstable manifold $\Gamma_{\delta, \varepsilon}^{u}$.

\section{Referências}

[1] B. Deng, Food chain chaos due to junction-fold point, Chaos, 11(3) (2001), 514-525.

[2] B. Deng and G. Hines, Food chain chaos due to Shilnikov's orbit, Chaos 12(3) (2002), $533-538$.

[3] N. Fenichel, Geometric singular perturbation theory for ordinary differential equations, J. Diff. Equations 31 (1979), 53-98.

[4] M. Krupa, N. Popović and N. Kopell Mixed-mode oscillations in three time-scale systems: a prototypical example, SIAM Appl. Dyn. Syst. 7(2) (2008), 361-420.

[5] W. Kunpasuruang, Y. Lenbury and G. Hek, A nonlinear mathmatical model for pulsatile discharges of luteinizing hormone mediated by hypothalamic and extra-hypothalamic pathways, Math Models Methods Appl. Sci. 12(5) (2002), 607-624.

[6] G.S. Medvedev and J.E. Cisternas, Multimodal regimes in a compartmental model of the dopamine neuron, Physica D 194(3-4) (2004), 333-356.

[7] S. Muratori and S. Rinaldi, Low- and high-frequency oscillations in three-dimensional food chain systems, SIAM J. Appl. Math. 52 (1992), 1688-1706.

[8] M.L. Rosenzweig and R.H. MacArthur, Graphical representation and stability conditions of predator-prey interactions, Am. Nat. 97 (1963), 209-223. 